\title{
The non-pathogenic Australian rabbit calicivirus RCV-A1 provides temporal and partial cross protection to lethal Rabbit Haemorrhagic Disease Virus infection which is not dependent on antibody titres
}

\author{
Tanja Strive ${ }^{1,2^{*}}$, Peter Elsworth ${ }^{2,3}$, June Liu ${ }^{1,2}$, John D Wright ${ }^{1,2}$, John Kovaliski ${ }^{2,4}$ and Lorenzo Capucci ${ }^{5}$
}

\begin{abstract}
The endemic non-pathogenic Australian rabbit calicivirus RCV-A1 is known to provide some cross protection to lethal infection with the closely related Rabbit Haemorrhagic Disease Virus (RHDV). Despite its obvious negative impacts on viral biocontrol of introduced European rabbits in Australia, little is known about the extent and mechanisms of this cross protection. In this study 46 rabbits from a colony naturally infected with RCV-A1 were exposed to RHDV. Survival rates and survival times did not correlate with titres of serum antibodies specific to RCV-A1 or cross reacting to RHDV, but were instead influenced by the time between infection with the two viruses, demonstrating for the first time that the cross protection to lethal RHDV infection is transient. These findings are an important step towards a better understanding of the complex interactions of co-occurring pathogenic and non-pathogenic lagoviruses.
\end{abstract}

\section{Introduction}

The prototype of the genus Lagovirus within the family Caliciviridae is Rabbit Haemorrhagic Disease Virus (RHDV) [1]. RHDV causes mortality rates of up to $90 \%$ in European rabbits (Oryctolagus cuniculus), the only species susceptible to the virus. It causes necrotizing hepatitis of the liver, severe disseminated intravascular coagulation and multiple organ failure, and usually kills rabbits within $72 \mathrm{~h}$ [2-4]. RHDV was first reported in an Angora rabbit colony in China [5], although recent phylogenetic analysis suggests that pathogenic RHDV may have evolved several decades earlier, also in Asia [6,7]. In the past 25 years RHDV has spread amongst domestic and wild rabbits across the world, causing economic losses to the meat industry [8] and ecological

\footnotetext{
* Correspondence: tanja.strive@csiro.au

${ }^{1}$ Division of Ecosystem Sciences, Commonwealth Scientific and Industrial Research Organisation, Canberra ACT 2601, Australia

${ }^{2}$ Invasive Animals Cooperative Research Centre, University of Canberra, Canberra ACT 2601, Australia

Full list of author information is available at the end of the article
}

damage in countries where wild rabbits are a vital part of the ecosystem [9].

Australia heavily relies on RHDV to control overabundant European rabbits that were introduced to the continent approximately 150 years ago and multiplied to plague proportions, causing severe damage to native vegetation and impacting on the meat and wool industry [10-12]. In 1996, RHDV was officially approved for release as a rabbit biocontrol agent in Australia [10], and was very successful in reducing rabbit numbers initially [13]. However, it did not kill rabbits very effectively in some areas of Australia, and in rabbits from these regions antibodies cross reacting to RHDV were found [14]. This lead to the hypothesis that related endemic caliciviruses were circulating in these rabbits, providing some level of cross protection to lethal RDHV infection $[15,16]$. Such a virus was recently identified in wild Australian rabbits [17] and was designated Rabbit Calicivirus Australia 1 (RCV-A1). Evolutionary analysis suggests that this virus arrived in Australia together with the first wild rabbits, approximately 150 years ago [18]. Pilot infection studies showed that RCV-A1 causes a non-pathogenic 
infection of the small intestine and is capable of providing partial cross protection to lethal RHDV infection [19], confirming RCV-A1 is hindering effective RHDV-mediated rabbit biocontrol.

$\mathrm{RCV}-\mathrm{A} 1$ adds to the growing number of non-pathogenic lagoviruses related to RHDV that are phylogenetically distinct from RHDV [20] and that have been reported from Italy [21], France [22-24], England [25,26] and moderately pathogenic viruses from the United States [27] and Europe [28]. Notably, studies that experimentally tested the immunological cross protection to RHDV conveyed by the non-pathogenic caliciviruses revealed disparate and partly unexpected results. A pilot infection study conducted with RCV-A1 resulted in 50\% surviving the RHDV challenge, although only very low numbers were used in this study $(n=4)$ [19]. In several earlier studies wildcaught Australian rabbits presumed to have antibodies to a then unidentified RCV-A1 were challenged with RHDV and the observed protection rates varied between 36\% [15], 33\% [29] and 52\% [14]. In contrast, the first non-pathogenic lagovirus that was described by Capucci et al. in Italy proved to be $100 \%$ protective to lethal RDHV infection [21]. Surprisingly, Le GallReculé et al. did not find any cross protection provided by a recently isolated non-pathogenic French lagovirus [23], although this virus is genetically much more similar to RHDV than the Australian RCV-A1. These divergent findings underline the need to better understand both the extent and the mechanisms of cross protection provided by non-pathogenic relatives of RHDV.

In addition, immunological cross protection contributes to the complex interplay between host and pathogen, and the processes of host-pathogen co-evolution. Australian rabbits are beginning to develop genetic resistance to RHDV [30], by mechanisms that are not completely understood. It has been shown that HistoBlood Group Antigens (HBGAs) on the epithelial linings of the rabbit gastro intestinal tract act as attachment factors for RHDV [31]. HBGA's are synthesised by the enzyme \#1,2 fucosyltransferase, which in rabbits is encoded by three functional genes, Fut1, Fut2, and Sec1 that have undergone multiple events of gene conversion during evolution [32]. Recent work suggests that different RHDV strains bind preferentially to different HBGA ligands, and rabbits expressing weaker binding HBGA phenotypes are found with increased frequency in wild rabbit populations following RHDV outbreaks [33,34]. Therefore, if a wild rabbit population is partially protected from lethal RHDV infection by immunological cross protection from a nonpathogenic calicivirus, this may reduce the selective pressure towards genetically resistant rabbits with weaker binding HBGA phenotypes as a means to avoid lethal RHDV infection [33].
Shedding more light on the extent and mechanisms of the immunological cross protection conveyed by the different non-pathogenic rabbit caliciviruses that are cocirculating with RHDV in wild rabbit populations is therefore vitally important to fully understand the implications for rabbit control in Australia. Conversely, such knowledge could contribute to the selection of nonpathogenic virus strains that could help protect endangered wild rabbit populations from RHDV outbreaks in Europe. While commercially available vaccine have proven effective in commercial rabbitries, vaccination campaigns in wild rabbits are economically and logistically impracticable [20].

The aim of this study was to gain better understanding of the extent of the cross protection that previous RCVA1 infection can confer to lethal RHDV challenge by exposing a large number $(n=46)$ of rabbits with RCV-A1 antibodies to RHDV. We further investigated if the cross protection is dependent on the titres of antibodies raised against RCV-A1 and cross reacting to RHDV. We furthermore assessed if the protection rates vary when animals are experimentally inoculated with RHDV or acquire the infection via contact transmission.

\section{Materials and methods}

\section{Animals and experimental design}

Fifty, twelve-week-old domestic rabbits were acquired from a commercial rabbit breeding facility where RCVA1 was circulating and individually marked with ear tags. Blood samples were taken at arrival (week 0), and then at week 3, 4 and 8 and analysed for antibodies against RHDV. One rabbit died shortly after arrival due to unknown causes, but unrelated to infection with either RCV-A1 or RHDV. Three of the rabbits that were either seronegative or IgM positive to antibodies cross reacting to RHDV at arrival were euthanazed two weeks after arrival. Their duodenum, liver and bile were analysed for the presence of lagoviruses using a universal lagovirus PCR that detects both RCV-A1 and RHDV, as described previously [17]. Male $(n=15)$ and female rabbits $(n=31)$ were housed separately in two group pens. Both pens had a floor area of approximately $15 \mathrm{~m}^{2}$. Eight weeks post acquisition 26 rabbits $(9$ males and $17 \mathrm{fe}-$ males) were infected orally with $500 \mathrm{LD}_{50}$ of a commercially available RHDV-preparation (Elizabeth McArthur Agricultural Institute, Menangle, Australia) and returned to the group-pens.

The aim of this study was to assess if cross protection is dependent on the titres of RCV-A1-induced antibodies cross reacting to RHDV, and if there is a difference depending on the infectious dose of RHDV received. Therefore, the rabbits were divided into two groups, one group was experimentally inoculated with a moderate dose of RHDV (500 LD $\mathrm{L}_{50}$ ), and the second was 
placed into the same pens to acquire the infection via contact (Table 1), such that both the experimentally infected rabbits and contact rabbits had high, medium or no IgG antibody titres cross reacting to RHDV at week 0 (Additional file 1). For the first $24 \mathrm{~h}$, infected and non-infected animals were kept separate by a divider in the group pen to avoid low-dose infection of the contact animals through inoculum-contamination on feeders and water bottles. On the second day the dividers were removed and infected and contact animals were allowed to mix again to allow true contact infection. Throughout the trial, rabbits had ad libitum access to oaten hay and commercial rabbit pellets, as well as water bottles and dishes.

Following the RHDV challenge, rectal temperatures were monitored twice daily and body weights were recorded on a daily basis. In addition, rabbits were checked every four hours during the day to record the time of death as accurately as possible. When found dead, rectal temperatures were taken and used to extrapolate the time of death based on experimentally determined post mortem temperature decay profiles (P. Elsworth, unpublished data). Where temperatures had dropped to near room temperature ( $>8 \mathrm{~h}$ post mortem), the time since death $\left(t_{d}\right)$ was calculated as time between when the rabbit was last seen alive and found dead $(\Delta t)$ minus half the difference between $\Delta \mathrm{t}$ and $8 \mathrm{~h}: \mathrm{t}_{\mathrm{d}}[\mathrm{h}]=\Delta \mathrm{t}-((\Delta \mathrm{t}[\mathrm{h}]-8) / 2)$. A final blood sample was collected from each animal at point of death, and at days 13 and 30 post RHDV challenge from the surviving rabbits, to monitor seroconversion to RHDV. The trial was terminated 30 days after the RHDV challenge and all remaining rabbits were euthanazed.

In order to assess if the observed survival rates and survival times were influenced by the duration between the infection with the two viruses, rabbits were divided into groups according to the time of their seroconversion to RCV-A1 for additional analysis. All rabbits that were seronegative or equivocal for RCV-A1 antibodies at week 0 , but seroconverted within the next three weeks $(n=8)$ were assigned to Group 1 ( $<8$ weeks between the RCV-A1 and RHDV infection). Group $2(n=8)$ contained animals that tested positive for RCV-A1 IgM antibodies at week 0 or 3 as well as one animal that tested negative for RCV-A1 IgG at week 0 but positive at week 3 . The time between infections for Group 2 was estimated based on previous observations that IgM antibodies to RCV-A1 appear from day 3 post infection (pi) and are usually detectable for at least 2 weeks $[19,35]$ (and unpublished data), the time of RCV-A1 infection of the IgM positive animals in group two was therefore inferred to have been approximately 8-10 weeks prior to RHDV challenge. This group also contained rabbits \#20 and \#50, who had anti-RCV-A1 IgM antibodies at week 3 although their serostatus at week 0 was uncertain (Additional file 1).
The third Group was formed by animals $(n=18)$ that were already seropositive when purchased and that had acquired the RCV-A1 infection at an unknown time before acquisition. The remaining animals $(n=12)$ that had lost their ear tags and showed no RCV-A1 IgM at any time were not included into analyses that required information about their time of seroconversion to RCV-A1 (Table 1), however they were included into analyses assessing possible correlations of serum antibody titres at the time of challenge with survival times and survival rates.

All procedures involving animals were carried out according to the "Australian Code of Practice for the Care and Use of Animals for Scientific Purposes" and were approved by the Australian Department of Agriculture, Fisheries and Forestry Community Access Animal Ethics Committee (\#CA 2008/09/303).

\section{Detection of viral RNA}

RNA was extracted using the RNEasy kit (Qiagen, Hilden, Germany) for tissue samples, and the Invitrogen PureLink viral RNA kit (Invitrogen, Melbourne, Australia) for serum and bile samples, as per the respective protocols provided by the suppliers. Established protocols were used for the universal lagovirus RT-PCR [17], and RHDV real time qRT-PCR [19]. RCV-A1 qRT-PCR was carried out as described previously [36], but with primers WAU-1 $1 \mathrm{~F}$ (ACCCTACAACCAACACATCAGG) and WAU-1 1R (ATGCCTGAAGCCAAAATAAACA). These primers are highly specific to the RCV-A1 virus strain used in this study and do not bind RHDV.

\section{Serology}

ELISAs were used for the detection of IgA, IgG and IgM antibodies to RHDV $[21,37,38]$ and RCV-A1 [39] as previously described. Sera were tested in duplicates, starting at a 1:40 dilution and subsequent 4 fold dilution steps. Sera that were only qualitatively tested to be either seropositive or negative were only analysed at a 1:40 and 1:160 dilution, also in duplicates. As the isotype ELISAs for the two viruses are known to cross react to various degrees, a more specific competition ELISA (cELISA) for RHDV and a specific blocking ELISA for RCV-A1 [40] were also used, starting at a 1:10 dilution with subsequent 4 fold dilution steps.

\section{Results}

Several attempts were made to purchase 50 seronegative, 12 week old un-vaccinated rabbits from vaccinated does. At the time the trial was conducted, no RCV-specific ELISAs was available, therefore assays originally developed for RHDV were used to assess the antibody status of the animals. The first batch was unsuitable as almost all rabbits had very high anti-RHDV antibody titres, indicating exposure to RHDV at a young age and subsequent 
Table 1 Experimental groups, mortality rates, survival times and fever responses of the 46 rabbits subjected to RHDV challenge following previous RCV-A1 exposure

\begin{tabular}{|c|c|c|c|c|c|c|c|}
\hline ID & Sex & Inoculation & Survival & Survival time $[\mathrm{d}]$ & Adjusted survival time $[d]^{*}$ & Fever** & Duration of fever $[\mathrm{h}]{ }^{* * *}$ \\
\hline \multicolumn{8}{|c|}{ Group 1} \\
\hline \multicolumn{8}{|c|}{$<8$ weeks between RCV-A1 and RHDV infection } \\
\hline 18 & $m$ & infected & $Y$ & & & $Y$ & \\
\hline 11 & $f$ & contact & $Y$ & & & $N$ & \\
\hline 40 & $f$ & contact & $Y$ & & & $Y$ & \\
\hline 7 & m & infected & $\mathrm{N}$ & 8.8 & & Y & 41.0 \\
\hline 29 & $\mathrm{~m}$ & infected & $\mathrm{N}$ & 9.9 & & Y & 5.0 \\
\hline 31 & $f$ & infected & $\mathrm{N}$ & 2.6 & & $\mathrm{~N}$ & \\
\hline 37 & m & infected & N & 11.4 & & Y & 15.0 \\
\hline 36 & $\mathrm{~m}$ & contact & $\mathrm{N}$ & 12.2 & 9.8 & Y & 13.0 \\
\hline \multicolumn{8}{|c|}{ Group 2} \\
\hline \multicolumn{8}{|c|}{ 8-10 weeks between RCV-A1 and RHDV infection } \\
\hline 25 & $f$ & contact & $Y$ & & & $Y$ & \\
\hline 20 & $\mathrm{~F}$ & infected & $\mathrm{N}$ & 10.5 & & Y & 14.0 \\
\hline 38 & $\mathrm{~m}$ & infected & $\mathrm{N}$ & 8.5 & & $\mathrm{~N}$ & \\
\hline 50 & f & infected & N & 4.5 & & Y & 14.5 \\
\hline 28 & $\mathrm{~m}$ & contact & $\mathrm{N}$ & 10.4 & 8.0 & Y & 15.0 \\
\hline 39 & $\mathrm{~m}$ & contact & $\mathrm{N}$ & 12.2 & 9.8 & Y & 4.5 \\
\hline 44 & $f$ & contact & $\mathrm{N}$ & 9.9 & 7.5 & Y & 15.0 \\
\hline 47 & $f$ & contact & $\mathrm{N}$ & 7.0 & 4.6 & Y & 22.0 \\
\hline Group & & & & & & & \\
\hline
\end{tabular}

\section{$>10$ weeks between RCV-A1 and RHDV infection}

\begin{tabular}{|c|c|c|c|c|c|c|c|}
\hline 32 & $f$ & infected & $Y$ & & & $Y$ & \\
\hline 2 & f & infected & $\mathrm{N}$ & 4.5 & & Y & 14.5 \\
\hline 3 & f & infected & $N$ & 3.4 & & Y & 2.3 \\
\hline 4 & f & infected & $N$ & 2.6 & & Y & 7.5 \\
\hline 6 & $\mathrm{~m}$ & infected & $N$ & 7.4 & & Y & 16.0 \\
\hline 12 & f & infected & $\mathrm{N}$ & 4.4 & & Y & 5.3 \\
\hline 15 & f & infected & $N$ & 4.6 & & Y & 15.8 \\
\hline 16 & $\mathrm{~m}$ & infected & $N$ & 7.3 & & Y & 14.0 \\
\hline 17 & $\mathrm{~m}$ & infected & $N$ & 8.3 & & Y & 6.5 \\
\hline 19 & $\mathrm{~m}$ & infected & $\mathrm{N}$ & 3.2 & & Y & 3.5 \\
\hline 21 & f & infected & $N$ & 2.5 & & Y & 14.0 \\
\hline 24 & f & infected & $N$ & 2.2 & & N & \\
\hline 8 & $\mathrm{~m}$ & contact & $N$ & 7.0 & 4.6 & Y & 5.0 \\
\hline 23 & f & contact & $N$ & 6.1 & 3.7 & Y & 4.5 \\
\hline 26 & $\mathrm{~m}$ & contact & $\mathrm{N}$ & 6.2 & 3.8 & Y & 9.0 \\
\hline 27 & $\mathrm{~m}$ & contact & $N$ & 9.3 & 6.9 & Y & 12.0 \\
\hline 33 & f & contact & $N$ & 11.0 & 8.6 & $N$ & \\
\hline 45 & $f$ & contact & $N$ & 4.2 & 1.8 & Y & 6.5 \\
\hline
\end{tabular}


Table 1 Experimental groups, mortality rates, survival times and fever responses of the $\mathbf{4 6}$ rabbits subjected to RHDV challenge following previous RCV-A1 exposure (Continued)

\begin{tabular}{|c|c|c|c|c|c|c|c|}
\hline \\
\hline \multicolumn{8}{|c|}{$30 \quad f \quad$ infected $\quad Y$} \\
\hline \multicolumn{7}{|c|}{$\begin{array}{lllll}10 & f & \text { infected } & \mathrm{N} & 6.2\end{array}$} & 6.5 \\
\hline \multirow{2}{*}{$\begin{array}{l}14 \\
35\end{array}$} & f & infected & $\mathrm{N}$ & \multicolumn{2}{|l|}{2.8} & Y & 11.5 \\
\hline & f & infected & N & \multicolumn{2}{|l|}{9.5} & Y & 32.0 \\
\hline \multirow{2}{*}{$\begin{array}{l}48 \\
49\end{array}$} & f & infected & N & \multicolumn{2}{|l|}{9.7} & Y & 11.0 \\
\hline & $f$ & infected & $\mathrm{N}$ & \multicolumn{2}{|l|}{4.0} & Y & 3.0 \\
\hline \multirow{2}{*}{$\begin{array}{l}1 \\
5\end{array}$} & f & contact & N & 7.8 & 5.4 & Y & 12.0 \\
\hline & f & contact & N & 7.7 & 5.3 & Y & 9.5 \\
\hline 34 & f & contact & $N$ & 15.8 & 13.4 & $N$ & \\
\hline 42 & f & contact & $N$ & 6.7 & 4.3 & Y & 9.5 \\
\hline 43 & f & contact & $N$ & 4.7 & 2.3 & $N$ & \\
\hline 46 & f & contact & $\mathrm{N}$ & 5.2 & 2.8 & Y & 5.0 \\
\hline
\end{tabular}

Surviving animals are shown in bold italics.

*Adjusted survival time is -2.4 days for the contact infected animals.

** Temperature $>40.5^{\circ} \mathrm{C}$. *** Time between recorded onset of fever and death.

seroconversion. The second batch had a high proportion (approx 60\%) of rabbits with cross reactive antibodies to a putative benign virus, as inferred using the methods described by Cooke et al. [38]. However, as it was not known which RCV-A1 strain had caused seroconversion in these rabbits, this cohort of rabbits was equally unsuitable. The third group of 50 rabbits also had antibodies cross reacting to RHDV, however 17 animals were still seronegative, and one had IgM antibodies cross reacting to RHDV, indicating that a benign virus infection was currently circulating in the colony. This final group of rabbits was then used to study the effects on RHDV infection in a colony naturally infected with RCV-A1.

Three of the rabbits that were either seronegative or had IgM antibodies cross reacting to RHDV at arrival (\#13, \#22 and \#41) were euthanazed at week 2 and their duodenum, liver and bile analysed for the presence of lagoviruses in an attempt to isolate the circulating RCVA1 strain. One rabbit (\#22) tested positive in the PCR, indicating an active infection with a previously unknown RCV-A1 strain. The new strain was designated RCV-A1 WAU-1. Its relationship to the originally published strain MIC 1-4 [17] has been described elsewhere [18]. One rabbit (\#9) died shortly after arrival due to unknown causes, but unrelated to infection with either RCV-A1 or RHDV, as no viral RNA for either virus could be detected in the duodenum or liver, respectively.

At the time of arrival (week 0), 14 of the 46 experimental rabbits tested negative or equivocal in all three RHDV ELISAs (IgG, IgM and cELISA), and all except 5 developed antibodies cross reacting to RHDV within 8 weeks following arrival. As soon as specific ELISAs for RCV-A1 were available $[39,40]$, the sera were re- analysed. Eight of the 46 animals were seronegative or equivocal for RCV-A1 IgG, IgA and IgM at week 0 , and by week 4 all rabbits had seroconverted to $\mathrm{RCV}-\mathrm{A} 1$ (Additional file 1). Unfortunately, 14 rabbits in the female group had lost their ear tags on the first day, after the initial blood sample was collected. Numbers were reassigned to these animals according to their bodyweights, however as we could not be entirely certain that the numbers were re-assigned correctly, no serology data is available for week 0 for these animals.

Eight weeks post acquisition, 26 of the rabbits were perorally challenged with RHDV (Table 1) and placed back into the group pens, so that the remaining rabbits could acquire the infection via contact. The overall survival rate was $13 \%$ (6 of 46), and three of the survivors had received RHDV via direct inoculation and three via contact infection. Fever was detected in $83 \%$ of all rabbits $(38 / 46)$, with $85 \%(34 / 40)$ of the non-survivors and $67 \%(4 / 6)$ of the surviving rabbits showing temperatures above $40.5^{\circ} \mathrm{C}$. The time to onset of fever varied greatly between individuals, the average time between the first detection of fever and death of the animals however was consistently short $(11.6 \pm 8 \mathrm{~h}($ Mean $\pm \mathrm{SD}))$ (Table 1$)$.

Five of the six surviving rabbits seroconverted to RHDV at the end of the trial (Table 2), as indicated by high titres in the RHDV cELISA. One rabbit (\#11) avoided infection with RHDV. The serum of this rabbit showed medium titres in the RHDV IgG ELISA, however in the absence of a positive RHDV c-ELISA, IgM and IgA result, the IgG titres are likely to be RCV-A1 antibodies cross reacting in the RHDV IgG ELISA. This rabbit also had no detectable fever response. Rabbit \#30 also showed no fever, however this rabbit was just 
Table 2 Serology (RHDV only) of rabbits surviving the RHDV challenge

\begin{tabular}{|c|c|c|c|c|c|c|c|c|c|c|c|c|}
\hline \multirow[b]{3}{*}{ ID } & \multirow[b]{3}{*}{ Sex } & \multirow[b]{3}{*}{ Inoculation } & \multirow{3}{*}{$\begin{array}{c}\text { Weight } \\
\text { loss* }\end{array}$} & \multicolumn{3}{|c|}{ Antibodies to RHDV } & & & & & & \multirow[t]{3}{*}{ RHDV copies/ $\mu$ l bile } \\
\hline & & & & \multicolumn{2}{|c|}{$\lg M$} & \multicolumn{2}{|c|}{$\lg A$} & \multicolumn{2}{|c|}{$\lg G$} & \multicolumn{2}{|c|}{ cELISA } & \\
\hline & & & & $13 \mathrm{dpc}$ & $30 \mathrm{dpc}$ & $13 \mathrm{dpc}$ & $30 \mathrm{dpc}$ & $13 \mathrm{dpc}$ & $30 \mathrm{dpc}$ & $13 \mathrm{dpc}$ & $30 \mathrm{dpc}$ & \\
\hline 18 & $\mathrm{~m}$ & infected & Y & + & - & + & + & $>40960$ & $>40960$ & $>640$ & $>640$ & $4.11 E+03$ \\
\hline 32 & $f$ & infected & Y & + & + & + & + & $>40960$ & $>40960$ & $>640$ & $>640$ & $1.55 \mathrm{E}+04$ \\
\hline 40 & $f$ & contact & $N$ & + & + & + & + & $\mathrm{D}$ & $>40960$ & 320 & $>640$ & $5.23 E+04$ \\
\hline 25 & $f$ & contact & N & - & - & - & $\mathrm{D}$ & 40 & $>40960$ & 20 & $>640$ & $2.07 \mathrm{E}+01$ \\
\hline 30 & $f$ & infected & N & - & - & - & + & 1280 & 5120 & - & 160 & $1.70 \mathrm{E}+01$ \\
\hline 11 & $f$ & contact & N & - & - & - & - & - & 1280 & - & - & - \\
\hline
\end{tabular}

Titres are expressed as the reciprocal dilutions at which the sera tested positive.

* Weight loss $>10 \%$, dpc $=$ days post RHDV challenge, $-=$ negative,$+=$ positive

$D$, Doubtful.

beginning to seroconvert to RHDV at day 30 post challenge (dpc), (Table 2). As temperatures were not monitored regularly beyond $13 \mathrm{dpc}$, a possible fever episode may therefore have not been detected in this rabbit. Of the six surviving rabbits two (\#18 and \#32) showed temporary weight loss $(>10 \%$ but $<20 \%$ ) following their fever episode; these were the only two cases of weight loss $>$ $10 \%$ of all 46 rabbits in this experiment. These two animals also had a clear, highly viscous mucous discharge from the rectum for one day following their fever episode.

Unexpectedly, no correlation was observed between survival rates or survival times and serum antibody titres at the time of RHDV challenge. Pearson's Product Moment Correlation revealed low but insignificant levels of negative correlation (RHDV-IgG: $\mathrm{t}=-1.54, p=0.1319$; RCV-A1-IgG: $\mathrm{t}=-1.4, \quad p=0.1696 ;$ RCV-A1-IgA: $\mathrm{t}=-0.914, p=0.3667$ and RCV-A1-bELISA: $\mathrm{t}=-0.568, p=0.5737)$. Similarly, there was no correlation between survival rates and any of the antibody titres (Chi-Squared test, data not shown).

Notably, the average survival times of all rabbits that died were unusually high, 7.1 days \pm 3.3 (Mean \pm SD). Survival times for contact-infected rabbits were longer (8.4 days \pm 3.1 (Mean \pm SD)) compared to experimentally inoculated rabbits (6.0 days \pm 3.1 (Mean \pm SD)). This 2.4 day average difference reflects the delay in acquiring the infection from the infected rabbits that were shedding virus.

Both the infected as well as the contact animals died in two cohorts. The first cohort died between day 2 and 4 in the infected animals and day 4 to 7 in the contact animals. The second cohort died between day 7 to 11 in the infected, and day 9 to 12 in the contact animals, respectively. When the survival times for the contact animals were corrected by the average time delay it took them to acquire the infection ( -2.4 days), a clear biphasic pattern was observed. Notably, the animals that died in the second cohort showed no signs of prolonged disease, but appeared healthy until they suddenly developed a fever and died.

A high proportion of rabbits that had been seronegative to either RHDV or RCV-A1 when purchased and subsequently seroconverted within the following 8 weeks died in the second wave. This prompted us to investigate if the time between the infections with RCV-A1 and RHDV had an influence on survival rates and survival times, and animals were grouped according to the time between the two infections (Figure 1 and Table 1). In the group of the recently seroconverted animals $(<8$ weeks between infections of RCV-A1 and RHDV) 3 of 8 rabbits survived (38\%) and the animals in this group that did not survive showed prolonged survival times, with a median survival time of 9.9 days. The median survival time in the second group of animals with 8-10 weeks between infections with the two viruses was 8.0 days, and only one rabbit survived (13\%). In comparison, median survival time in animals with a mature immune response in group three was 4.4 days, and in this group too only one animal survived (6\%). In the Kaplan Meier survival analysis, the Logrank test was highly significant between group 1 and group $3(p=0.002)$ and significant between group 2 and group $3(p=0.038)$, but not significant between group 1 and group 2 .

All rabbits that succumbed to RHDV infection had high levels of RHDV RNA in their bile $\left(10^{5}\right.$ to $10^{9}$ copies per microliter) as determined by qRT-PCR, confirming RHDV as the likely cause of death (data not shown). Of the six surviving rabbits three had moderate viral loads of RHDV in the bile, while rabbits \#25 and \#30 had very low amounts of viral RNA just above the detection threshold, and rabbit \#11 had no detectable RHDV RNA in the bile (Table 2). Trace amounts of RCV-A1 RNA just above the detection limit $\left(1.4 \times 10^{1}\right.$ copies per microliter) were detected in the bile of rabbit \#37 (data not shown), all other rabbits tested negative for RCV-A1 at time of death or euthanasia.

\section{Discussion}

The most surprising finding of this study was that the partial protection provided by RCV-A1 to lethal RHDV infection does not depend on the titres of antibodies 


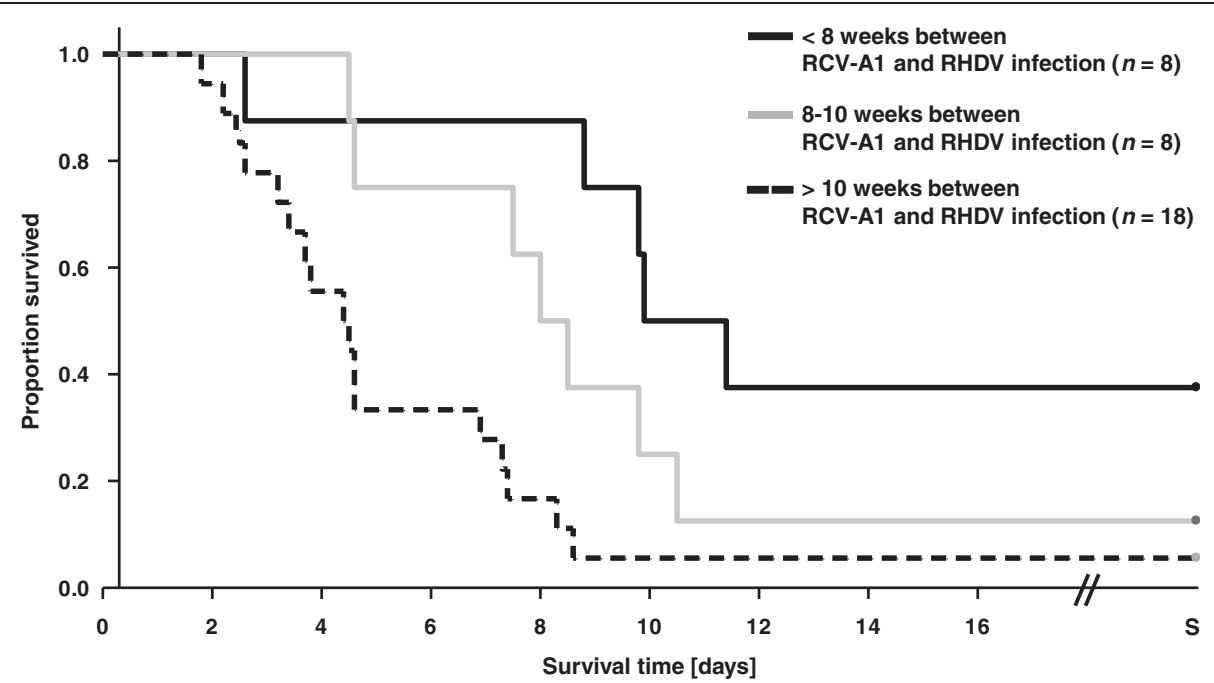

Figure 1 Kaplan Meier survival analysis of rabbits challenged with RHDV. Analysis was carried out using the SigmaPlot software version 12.3. S indicates survival until 30 days post RHDV challenge when the rabbits were euthanazed.

cross reacting to RHDV. This observation differs from results obtained in previous pilot infection studies, where the survival rate following RCV-A1 infection was $50 \%$, and the surviving animals had higher antibody titres cross reacting to RHDV compared to the nonsurvivors [19]. However in that study only a small number of animals was used $(n=4)$. The data presented here show that the titres of serum antibodies both specific to RCV-A1 and cross reacting to RHDV are poor predictors for survival.

Instead, our results indicate that the timing of the RCV-A1 infection is likely to play a major role in the outcome of an RHDV challenge. There was a marked difference in the survival rates and survival times depending on the time between RCV-A1 and RHDV infection. As it was not known when the rabbits that were already seropositive at week 0 had acquired their RCVA1 infection and serum samples were only collected at week 3,4 , and 8 , it was only possible to assign the rabbits to three groups (Group 1: $<8$ weeks; Group 2: 810 weeks and Group 3: > 10 weeks). Our results show that Group 1 rabbits with no more than 8 weeks between infections with the two viruses had the highest survival rates (38\%) and longest survival times (median 9.9 days). Protection rates decreased to $13 \%$ in Group 2, while the survival times in this group were still unusually long with a median of 8 days. In contrast, for Group 3 rabbits with a mature immune response to RCV-A1 and at least 10 weeks between infections with the two viruses, there was no detectable protective effect against lethal RHDV infection. Survival rates of $6 \%$ and median survival times of 4.4 days are comparable to previous reports describing RHDV infections in naïve rabbits [2].
The temporal nature of the cross protection observed for RCV-A1 may help to explain the wide variations described for protection rates that the various nonpathogenic caliciviruses provide to RHDV challenge. Capucci et al. described complete protection (16/16 survived) when rabbits were challenged with RHDV, 24 days after infection with the Italian RCV [21]. Fifty percent protection (2/4 survived) was observed when rabbits were challenged with RHDV 24 days after infection with the Australian RCV-A1 [19] (and Table 3), and this reduced protection rate was initially ascribed to the lower degree of genetic relatedness and amino acid homologies to RHDV. Interestingly, Le-Gall and colleagues found no protection from RHDV challenge (1/15 survived) following previous infection with a recently discovered French non-pathogenic virus strain, although this European isolate is genetically more closely related to RHDV than RCV-A1 [23]. However, the rabbits subjected to RHDV challenge in their study were deliberately selected to exhibit high antibody titres cross reacting to RHDV, indicating mature antibody responses. Indeed, several of their rabbits had already been seropositive when acquired 3 weeks prior to challenge [23]. Our findings suggest that the window of heightened resistance to lethal RHDV infection may have been missed in their study. Future studies investigating the interference of pathogenic and non-pathogenic lagoviruses should take into account the time between infections as an important contributing factor to the varying degrees of cross protection.

As the timing of previous $\mathrm{RCV}-\mathrm{A} 1$ infection appears crucial for the outcome of subsequent RHDV challenge, an accurate estimate of overall protection rates conveyed 
Table 3 Summary of experiments assessing the level of cross protection to lethal RHDV infection provided by RCV-A1

\begin{tabular}{|c|c|c|c|c|c|c|}
\hline Study No. & Protection rate & $\mathrm{N}$ & RCV-A1 infection & $\begin{array}{l}\text { Time post RCV-A1 } \\
\text { infection }\end{array}$ & RHDV challenge & Reference \\
\hline 1 & $52 \%$ & $11 / 21$ & Natural infection & Unknown & One dose 1000 LD ${ }_{50}, \mathrm{IM}$ & [14] \\
\hline 2 & $36 \%$ & $22 / 61$ & Natural infection & Unknown & One dose 1500 LD $50, \mathrm{PO}$ & [15] \\
\hline 3 & $33 \%$ & $25 / 77$ & Natural infection & Unknown & One dose 1500 LD $500, \mathrm{PO}$ & [29] \\
\hline 4 & $50 \%$ & $2 / 4$ & $\begin{array}{l}\text { Experimental } \\
\text { infection }\end{array}$ & 4 weeks & One dose 500 LD ${ }_{50}, \mathrm{PO}$ & [19] \\
\hline $5 a$ & $38 \%$ & $3 / 8$ & Natural infection & $<8$ weeks & $\begin{array}{l}\text { One dose } 500 \text { LD }{ }_{50}, \mathrm{PO} \text { and/or continuous } \\
\text { exposure (contaminated environment) }\end{array}$ & This report \\
\hline $5 b$ & $13 \%$ & $1 / 8$ & Natural infection & 8 - 10 weeks & $\begin{array}{l}\text { One dose } 500 \text { LD }{ }_{50,}, \mathrm{PO} \text { and/or continuous } \\
\text { exposure (contaminated environment) }\end{array}$ & This report \\
\hline $5 c$ & $6 \%$ & $1 / 18$ & Natural infection & $>10$ weeks & $\begin{array}{l}\text { One dose } 500 \mathrm{LD}_{50}, \mathrm{PO} \text { and/or continuous } \\
\text { exposure (contaminated environment) }\end{array}$ & This report \\
\hline
\end{tabular}

IM, Intra muscular, PO, Peroral.

by RCV-A1 is difficult to make. In addition, our data and previously published observations suggest that a variety of factors, such as the dose of RHDV, may play an additional role in the outcome of RHDV challenge. Table 3 provides a comparison of the current results with previously published data on experimental RHDV challenges of rabbits that were either known to be previously infected with RCV-A1, or inferred to be RCV-A1 positive due to antibodies cross reacting to RHDV. Protection rates of $33 \%$ to $52 \%$ were observed in animals experimentally infected with one dose of RHDV, ranging from 500 to 1500 LD50, applied via the PO or IM route (Table 3, study 1-4) $[14,15,19,29]$. In contrast, the current study (Table 3, study 5a, 5b and 5c) was conducted as a pen trial where rabbits were housed in large groups, and were both experimentally infected and acquired the infection via contact. It has been shown that high amounts of RHDV-RNA can be detected in rabbit faeces shortly before they die [19], and it is therefore likely that in this environment, over time, rabbits were exposed to continuously increasing quantities of virus due to virus shed by animals that succumbed to the challenge. In this trial, water dishes in particular appear to be a likely source of continuous exposure, as all rabbits used them and, although the water was changed daily, they were heavily soiled with faecal material.

It is unclear at this stage if the rabbits with the unusually long survival times avoided infection or disease. For example, rabbits \#7, \#17, \#20, \#37, \#38 and \#48 died between days 8 and 11 pi. As these animals were in the group of experimentally infected animals, the exact time between experimental infection and death was known. From day 3 post challenge onwards, these animals should have had a developing antibody response to RHDV. No increases in antibody titres reacting to RHDV were detected in these animals at the time of death (data not shown), however the viral load in the serum and bile was very high $\left(10^{9}\right.$ to $10^{11}$ and $10^{5}$ to $10^{9}$ RNA copies $/ \mathrm{mL}$, respectively) as determined by qRTPCR (data not shown). It is possible that this high viremia neutralised moderate titres of early serum antibodies, resulting in negative ELISA results, although a successful adaptive response to RHDV usually results in effective clearance of infection. Alternatively, the initial experimental infection was unsuccessful and the rabbits acquired the virus later from the contaminated environment. Mucosal antibodies may have played a role in avoiding the infection but were not assessed in this study.

More research is needed to better understand the immunological mechanisms responsible for the temporal nature of the cross protection. Short-term elevation of non-specific innate immune mechanisms following RCVA1 infection may lead to increased levels of infectious disease resistance in general [41]. Alternatively, more mature immune responses to $\mathrm{RCV}$-A1 may be less protective to RHDV, due to affinity maturation. The selection of B-cells for production of antibodies with a higher affinity to the antigen (in this case RCV-A1) would lead to antibodies that are less cross reactive and thus possibly less protective to RHDV. However, the observation that antibody titres cross reacting to RHDV did not correlate with survival time or survival rates argues against this explanation.

Cellular immune mechanisms, which are known to be transiently elevated following infection, may also play an important role [41]. The $\mathrm{S}$ domain of the capsid protein VP60 that forms the inner shell of the viral particle [42] is highly conserved amongst lagomorph caliciviruses. RHDV and RCV-A1 have 93\% amino acid identity in their inner shell domains, but only $73 \%$ amino acid identity in the antigenic P2 domains. It is likely that some antigens that are presented as MHC-I complexes are derived from the conserved S-domain of the capsid protein VP60 or from other non-structural proteins. The 
resulting cross reacting T-cell receptors may recognise RHDV infected cells and contribute to temporary suppression of productive RHDV infection, resulting in increased survival times. In some cases the infection may be slowed down sufficiently for the rabbit to develop a strong adaptive immune response specific to RHDV, leading to clearance of infection and ultimately survival. Similar mechanisms have been suggested for Influenza virus, where CD8+ T-cell responses following natural infection with Influenza A/H3N2 can induce heterosubtypic protection to avian influenza A/H5N1 [43].

The importance of $\mathrm{T}$-cell responses in the protection from lethal RHD has also been discussed in the context of RHDV-vaccines, using RHDV specific antigens. Protection from VP60 expressed by a recombinant canarypox virus [44] or a recombinant ORF virus [45] to lethal RHDV was similarly independent from the vaccine induced antibody titres, and it was suggested that T-cell mediated immunity plays an important role [45]. However, commercial vaccines against RHDV that are based on inactivated, replication incompetent virus are effective in protecting rabbits against lethal RHD, and similarly recombinant VLPs also provide effective protection [46], although it has been demonstrated that VLPs alone are not effective in promoting the priming of naïve T-cells $[47,48]$. In addition, another study shows that adoptive transfer of polyclonal RHDV-positive serum can prevent lethal RHD in susceptible adult rabbits [49]. Future studies are clearly warranted shedding more light into the respective parts that cellular immunity, antibodies, but also immunogenetics [50] play in the protection and cross protection from lethal RHDV infection.

An additional intriguing result of this infection study was that it proved difficult to source seronegative rabbits that were devoid of antibodies cross reacting to RHDV due to a previous infection with benign calicivirus. Similar to murine norovirus, a calicivirus infecting mice that is non-pathogenic in the immunocompetent host and that was shown to be highly prevalent in scientific mouse breeding facilities tested [51], RCV-A1 may also be widespread amongst commercial rabbit breeding colonies. Non-pathogenic lagoviruses have been reported in European rabbitries [23], and more systematic testing of Australian rabbitries is clearly warranted to assess the prevalence of RCV-A1 in rabbits bred both for scientific purposes and for meat. Rabbit colonies may serve as a reservoir for RCV-A1, and transporting farmed rabbits or depositing soiled bedding material outside the breeding facilities is a potential mechanism facilitating the spread of RCV-A1.

The findings presented here have potentially important implications for rabbit biocontrol in Australia. The temporal nature of the cross protection conveyed by RCVA1 indicates that there may be a window of opportunity for RHDV to work effectively as a biocontrol agent, provided that RCV-A1 is not present throughout the year. Studies are therefore needed to determine the infection dynamics and seasonal occurrence of RCV-A1 in Australia. In contrast, if non-pathogenic caliciviruses were to be considered as natural vaccines to RHDV in countries where rabbits are a valued wildlife species [9], the timing of the release of such strains would be crucial to ensure maximum benefits to biodiversity conservation.

\section{Additional file}

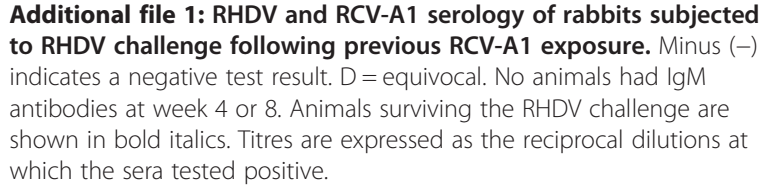

Competing interests

The authors declare they have no competing interests.

\section{Authors' contributions}

TS designed the study, wrote the manuscript and participated in conducting the animal experiments. PE participated in the design of the study, and conducted the animal experiments and statistical analysis. JL carried out and interpreted RCV-A1 immuno-assays. JDW carried out RCV-A1 immunoassays and participated in conducting the animal experiments. JK carried out RHDV immunoassays. LC participated in the study design, interpretation of results and provided key reagents. All authors have read and approved the final manuscript.

\section{Acknowledgements}

We would like to thank Marlene Jahnke, David Aster and Brian Koina for help with animal husbandry and monitoring, Divya George for technical assistance with qRT-PCR diagnostic, Brian Cooke for providing valuable advice on the study design, and Lyn Hinds and Peter Kerr for helpful comments on the manuscript.

\section{Author details}

${ }^{1}$ Division of Ecosystem Sciences, Commonwealth Scientific and Industrial Research Organisation, Canberra ACT 2601, Australia. ${ }^{2}$ Invasive Animals Cooperative Research Centre, University of Canberra, Canberra ACT 2601, Australia. ${ }^{3}$ Department of Agriculture, Fisheries and Forestry, Robert Wicks Pest Animals Research Centre, Biosecurity Queensland, Toowoomba 4350, QLD, Australia. ${ }^{4}$ Natural Resources Management Biosecurity Unit, Biosecurity South Australia, Adelaide, SA, Australia. ${ }^{5}$ Institutto Zooprofilattico Sperimentale della Lombardia e dell' Emilia Romagna, 25124, Brescia, Italy.

Received: 21 November 2012 Accepted: 12 June 2013

Published: 8 July 2013

\section{References}

1. Green KY, Ando T, Balayan MS, Berke T, Clarke IN, Estes MK, Matson DO, Nakata S, Neill JD, Studdert MJ, Thiel HJ: Taxonomy of the caliciviruses. $J$ Infect Dis 2000, 181(Suppl 2):S322-S330.

2. Ohlinger VF, Haas B, Thiel HJ: Rabbit hemorrhagic disease (RHD): characterization of the causative calicivirus. Vet Res 1993, 24:103-116.

3. Ward VK, Cooke BD, Strive T: Rabbit hemorrhagic disease virus and other lagoviruses. In Caliciviruses: Molecular and Cellular Virology. Edited by Hansman KGG. Norfolk, UK: Caister Academic Press; 2010:223-245.

4. Abrantes J, van der Loo W, Le Pendu J, Esteves PJ: Rabbit haemorrhagic disease (RHD) and rabbit haemorrhagic disease virus (RHDV): a review. Vet Res 2012, 43:12.

5. Liu SJ, Xue HP, Pu BQ, Qian NH: A new viral disease in rabbits. Anim Husb Vet Med 1984, 6:253-255. 
6. Duffy S, Shackelton LA, Holmes EC: Rates of evolutionary change in viruses: patterns and determinants. Nat Rev Genet 2008, 9:267-276.

7. Kerr PJ, Kitchen A, Holmes EC: Origin and phylodynamics of rabbit hemorrhagic disease virus. J Virol 2009, 83:12129-12138.

8. McIntosh MT, Behan SC, Mohamed FM, Lu Z, Moran KE, Burrage TG, Neilan JG, Ward GB, Botti G, Capucci L, Metwally SA: A pandemic strain of calicivirus threatens rabbit industries in the Americas. Virol J 2007, 4:96.

9. Delibes-Mateos M, Redpath SM, Angulo E, Ferrerasa P, Villafuerte R: Rabbits as a keystone species in southern Europe. Biol Conserv 2007, 137:149-156.

10. Cooke BD, Fenner F: Rabbit haemorrhagic disease and the biological control of wild rabbits, Oryctolagus cuniculus, in Australia and New Zealand. Wildlife Res 2002, 29:689-706.

11. Cooke BD: Rabbits: manageable environmental pests or participants in new Australian ecosystems? Wildlife Res 2012, 39:279-289.

12. Mutze G, Kovaliski J, Butler K, Capucci L, McPhee S: The effect of rabbit population control programmes on the impact of rabbit haemorrhagic disease in south-eastern Australia. J Appl Ecol 2010, 47:1137-1146.

13. Mutze $G$, Cooke $B$, Alexander $P$ : The initial impact of rabbit hemorrhagic disease on European rabbit populations in South Australia. J Wildl Dis 1998, 34:221-227.

14. Nagesha HS, McColl KA, Collins BJ, Morrissy CJ, Wang LF, Westbury HA: The presence of cross-reactive antibodies to rabbit haemorrhagic disease virus in Australian wild rabbits prior to the escape of virus from quarantine. Arch Virol 2000, 145:749-757.

15. Cooke BD, McPhee S, Robinson AJ, Capucci L: Rabbit haemorrhagic disease: does a pre-existing RHDV-like virus reduce the effectiveness of RHD as a biological control in Australia? Wildlife Res 2002, 29:673-682.

16. Robinson AJ, Kirkland PD, Forrester RI, Capucci L, Cooke BD, Philbey AW: Serological evidence for the presence of a calicivirus in Australian wild rabbits, Oryctolagus cuniculus, before the introduction of rabbit haemorrhagic disease virus (RHDV): its potential influence on the specificity of a competitive ELISA for RHDV. Wildlife Res 2002, 29:655-662.

17. Strive T, Wright JD, Robinson AJ: Identification and partial characterisation of a new lagovirus in Australian wild rabbits. Virology 2009, 384:97-105.

18. Jahnke M, Holmes EC, Kerr PJ, Wright JD, Strive T: Evolution and Phylogeography of the Nonpathogenic Calicivirus RCV-A1 in Wild Rabbits in Australia. J Virol 2010, 84:12397-12404.

19. Strive T, Wright J, Kovaliski J, Botti G, Capucci L: The non-pathogenic Australian lagovirus RCV-A1 causes a prolonged infection and elicits partial cross-protection to rabbit haemorrhagic disease virus. Virology 2010, 398:125-134.

20. Abrantes J, Esteves PJ: Not-So-Novel Michigan Rabbit Calicivirus. Emerg Infect Dis 2010, 16:1331-1332

21. Capucci L, Fusi P, Lavazza A, Pacciarini ML, Rossi C: Detection and preliminary characterization of a new rabbit calicivirus related to rabbit hemorrhagic disease virus but nonpathogenic. J Virol 1996 70:8614-8623.

22. Le Gall-Reculé G, Zwingelstein F, Boucher S, Le Normand B, Plassiart G, Portejoie Y, Decors A, Bertagnoli S, Guerin JL, Marchandeau S: Detection of a new variant of rabbit haemorrhagic disease virus in France. Vet Rec 2011, 168:137-138.

23. Le Gall-Reculé G, Zwingelstein F, Fages MP, Bertagnoli S, Gelfi J, Aubineau J, Roobrouck A, Botti G, Lavazza A, Marchandeau S: Characterisation of a non-pathogenic and non-protective infectious rabbit lagovirus related to RHDV. Virology 2011, 410:395-402.

24. Marchandeau S, Le Gall-Reculé G, Bertagnoli S, Aubineau J, Botti G, Lavazza A: Serological evidence for a non-protective RHDV-like virus. Vet Res 2005, 36:53-62.

25. Forrester NL, Boag B, Buckley A, Moureau G, Gould EA: Co-circulation of widely disparate strains of rabbit haemorrhagic disease virus could explain localised epidemicity in the United Kingdom. Virology 2009, 393:42-48.

26. Forrester NL, Trout RC, Gould EA: Benign circulation of rabbit haemorrhagic disease virus on Lambay Island, Eire. Virology 2007, 358:18-22.

27. Bergin IL, Wise AG, Bolin SR, Mullaney TP, Kiupel M, Maes RK: Novel calicivirus identified in rabbits, Michigan, USA. Emerg Infect Dis 2009, 15:1955-1962

28. Dalton KP, Nicieza I, Balseiro A, Muguerza MA, Rosell JM, Casais R, Alvarez $A L$, Parra F: Variant rabbit hemorrhagic disease virus in young rabbits, Spain. Emerg Infect Dis 2012, 18:2009-2012.
29. McPhee SR, Butler KL, Kovaliski J, Mutze G, Capucci L, Cooke BD: Antibody status and survival of Australian wild rabbits challenged with rabbit haemorrhagic disease virus. Wildlife Res 2009, 36:447-456.

30. Elsworth PG, Kovaliski J, Cooke BD: Rabbit haemorrhagic disease: are Australian rabbits (Oryctolagus cuniculus) evolving resistance to infection with Czech CAPM 351 RHDV? Epidemiol Infect 2012, 140:1972-1981.

31. Ruvoën-Clouet N, Ganière JP, André-Fontaine G, Blanchard D, Le Pendu J: Binding of rabbit hemorrhagic disease virus to antigens of the $\mathrm{ABH}$ histo-blood group family. J Virol 2000, 74:11950-11954.

32. Abrantes J, Posada D, Guillon P, Esteves PJ, Le Pendu J: Widespread gene conversion of alpha-2-fucosyltransferase genes in mammals. J Mol Evol 2009, 69:22-31.

33. Nyström K, Le Gall-Reculé G, Grassi P, Abrantes J, Ruvoën-Clouet N, Le Moullac-Vaidye B, Lopes AM, Esteves PJ, Strive T, Marchandeau S, Dell A, Haslam SM, Le Pendu J: Histo-blood group antigens act as attachment factors of rabbit hemorrhagic disease virus infection in a virus straindependent manner. PLoS Pathog 2011, 7:1-22.

34. Guillon P, Ruvoën-Clouet N, Le Moullac-Vaidye B, Marchandeau S, Le Pendu J: Association between expression of the $\mathrm{H}$ histo-blood group antigen, alpha 1,2fucosyltransferases polymorphism of wild rabbits, and sensitivity to rabbit hemorrhagic disease virus. Glycobiology 2009, 19:21-28.

35. Capucci L, Nardin A, Lavazza A: Seroconversion in an industrial unit of rabbits infected with a non-pathogenic rabbit haemorrhagic disease-like virus. Vet Rec 1997, 140:647-650.

36. Hoehn M, Kerr PJ, Strive T: In situ hybridisation assay for localisation of rabbit calicivirus Australia-1 (RCV-A1) in European rabbit (Oryctolagus cuniculus) tissues. J Virol Methods 2012, 188:148-152.

37. Capucci L, Scicluna MT, Lavazza A: Diagnosis of viral haemorrhagic disease of rabbits and the European brown hare syndrome. Rev Sci Tech 1991, 10:347-370.

38. Cooke BD, Robinson AJ, Merchant JC, Nardin A, Capucci L: Use of ELISAs in field studies of rabbit haemorrhagic disease (RHD) in Australia. Epidemiol Infect 2000, 124:563-576.

39. Liu J, Kerr PJ, Wright JD, Strive T: Serological assays to discriminate rabbit haemorrhagic disease virus from Australian non-pathogenic rabbit calicivirus. Vet Microbiol 2012, 157:345-354.

40. Liu J, Kerr PJ, Strive T: A sensitive and specific blocking ELISA for the detection of rabbit calicivirus RCV-A1 antibodies. Virol J 2012, 9:182.

41. Nikolich-Zugich J: Ageing and life-long maintenance of T-cell subsets in the face of latent persistent infections. Nature Rev Immunol 2008, 8:512-522.

42. Chen R, Neill JD, Noel JS, Hutson AM, Glass RI, Estes MK, Prasad BV: Interand intragenus structural variations in caliciviruses and their functional implications. J Virol 2004, 78:6469-6479

43. Bodewes R, Fraaij PL, Kreijtz JH, Geelhoed-Mieras MM, Fouchier RA Osterhaus AD, Rimmelzwaan GF: Annual influenza vaccination affects the development of heterosubtypic immunity. Vaccine 2012, 30:7407-7410

44. Fischer L, LeGros FX, Mason PW, Paoletti E: A recombinant canarypox virus protects rabbits against a lethal rabbit hemorrhagic disease virus (RHDV) challenge. Vaccine 1997, 15:90-96.

45. Rohde J, Schirrmeier H, Granzow H, Rziha HJ: A new recombinant Orf virus (ORFV, Parapoxvirus) protects rabbits against lethal infection with rabbit hemorrhagic disease virus (RHDV). Vaccine 2011, 29:9256-9264.

46. Laurent S, Vautherot JF, Madelaine MF, LeGall G, Rasschaert D: Recombinant rabbit hemorrhagic-disease virus capsid protein expressed in baculovirus self-assembles into virus-like particles and induces protection. J Virol 1994, 68:6794-6798

47. McKee SJ, Young VL, Clow F, Hayman CM, Baird MA, Hermans IF, Young SL, Ward VK: Virus-like particles and alpha-galactosylceramide form a selfadjuvanting composite particle that elicits anti-tumor responses. J Control Release 2012, 159:338-345.

48. Peacey M, Wilson S, Perret $R$, Ronchese F, Ward VK, Young V, Young SL, Baird MA: Virus-like particles from rabbit hemorrhagic disease virus can induce an anti-tumor response. Vaccine 2008, 26:5334-5337.

49. Ferreira $P G$, Dinis $M$, Costa ES, Aguas AP: Adult rabbits acquire resistance to lethal calicivirus infection by adoptive transfer of sera from infected young rabbits. Vet Immunol Immunopathol 2008, 121:364-369.

50. Pinheiro A, Lanning D, Alves PC, Mage RG, Knight $\mathrm{KL}$, van der Loo W, Esteves PJ: Molecular bases of genetic diversity and evolution of the 
immunoglobulin heavy chain variable region (IGHV) gene locus in leporids. Immunogenetics 2011, 63:397-408.

51. Hsu CC, Wobus CE, Steffen EK, Riley LK, Livingston RS: Development of a microsphere-based serologic multiplexed fluorescent immunoassay and a reverse transcriptase PCR assay to detect murine norovirus 1 infection in mice. Clin Diag Lab Immunol 2005, 12:1145-1151.

doi:10.1186/1297-9716-44-51

Cite this article as: Strive et al:: The non-pathogenic Australian rabbit calicivirus RCV-A1 provides temporal and partial cross protection to lethal Rabbit Haemorrhagic Disease Virus infection which is not dependent on antibody titres. Veterinary Research 2013 44:51.

\section{Submit your next manuscript to BioMed Central and take full advantage of:}

- Convenient online submission

- Thorough peer review

- No space constraints or color figure charges

- Immediate publication on acceptance

- Inclusion in PubMed, CAS, Scopus and Google Scholar

- Research which is freely available for redistribution 